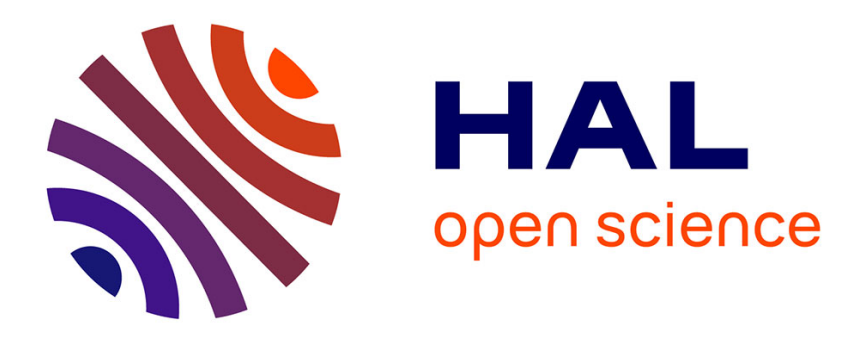

\title{
Aspects préliminaires de la théorie des machines électriques comportant des matériaux ferro-magnétiques \\ C. Rioux
}

\section{To cite this version:}

C. Rioux. Aspects préliminaires de la théorie des machines électriques comportant des matériaux ferro-magnétiques. Revue de Physique Appliquée, 1980, 15 (10), pp.1505-1515. 10.1051/rphysap:0198000150100150500 . jpa-00244875

\section{HAL Id: jpa-00244875 https://hal.science/jpa-00244875}

Submitted on 1 Jan 1980

HAL is a multi-disciplinary open access archive for the deposit and dissemination of scientific research documents, whether they are published or not. The documents may come from teaching and research institutions in France or abroad, or from public or private research centers.
L'archive ouverte pluridisciplinaire HAL, est destinée au dépôt et à la diffusion de documents scientifiques de niveau recherche, publiés ou non, émanant des établissements d'enseignement et de recherche français ou étrangers, des laboratoires publics ou privés. 


\title{
Aspects préliminaires de la théorie des machines électriques comportant des matériaux ferro-magnétiques
}

\author{
C. Rioux \\ Laboratoire d'Electrotechnique des Universités Paris VI et XI, Bâtiment 214, 91405 Orsay, France
}

(Reçu le 23 mai 1980, révisé le 30 juin 1980, accepté le 4 juillet 1980)

\begin{abstract}
Résumé. - L'étude examine les couplages électromécaniques qui prennent naissance dans les machines électriques comportant des matériaux magnétiques. Elle admet quelques hypothèses simples : la perméabilité ne dépend pas du champ et la structure est globalement de révolution, les écarts étant traités comme des perturbations linéaires. On définit ainsi deux échelles de longueurs caractéristiques : l'échelle globale liée aux dimensions des bobines et l'échelle locale donnée par les anisotropies de la distribution des matériaux magnétiques. On en déduit les structures globales que doivent avoir les machines classiques (utilisant les phénomènes aux échelles globales seules) et les machines à réluctance (qui utilisent les couplages entre les deux échelles).
\end{abstract}

\begin{abstract}
One studies the electromechanical couplings in machines with magnetic materials. A few simple assumptions are made : the permeability does not depends upon the magnetic field and the structure is in first approximation axially symmetric, linear perturbations being superimposed. Two scales of caracteristic lengths are defined : the global scale connected with the coils dimensions and the local scale given by the anisotropics of the magnetic materials distribution. One deduces the global structures required for the classical machines, which only utilize the global scale phenomena, and those for the reluctance machines which utilize the couplings between the two scales.
\end{abstract}

1. Introduction. - La présente étude examine de manière générale les couplages électromécaniques qui prennent naissance dans les machines électriques utilisant des matériaux magnétiques doux. Les objectifs de ce travail sont limités par le caractère restrictif des hypothèses de linéarisation admises :

- les matériaux employés ne sont pas saturables, c'est-à-dire que leur perméabilité est supposée indépendante du champ local dans lequel ils sont plongés (la perméabilité, par contre, peut être variable spatialement),

- les structures mécaniques et magnétiques des machines sont supposées globalement de révolution,

- si l'on creuse des cavités dans la structure globalement de révolution ci-dessus, leurs différentes influences sur les distributions des champs (notamment au niveau des entrefers) sont indépendantes les unes des autres.

Dans ce cadre d'approximations, on se propose de décrire les machines électriques ne comportant que des conducteurs et des matériaux magnétiques.

A cet effet, on donne tout d'abord les expressions du couple d'interaction rotor-stator issues du tenseur de Maxwell [1] ainsi que la distribution modale des champs dans le vide obtenus à partir de courants quelconques.

En utilisant le champ dans le vide comme source, on décrit le champ total en présence des matériaux magnétiques. On en déduit successivement :

- les différentes distributions engendrées,

- une forme linéarisée des équations du champ valable pour des cavités peu importantes,

- la portée des perturbations du champ engendrées par la présence locale d'une cavité.

A partir de ces éléments, on effectue alors un premier classement des machines qui fait apparaître trois types :

- les machines classiques,

- les machines à réluctance variable,

- les machines mixtes (combinaison peu importante des deux types précédents).

On montre qu'il existe dans les machines deux échelles de longueur : une échelle globale caractérisant les dimensions des bobinages et une échelle locale caractéristique des distributions de cavités. A l'aide de ces échelles on définit deux couches actives, relatives aux courants et aux cavités, qui permettent de compa- 
rer entre eux les couplages électromécaniques obtenus dans les différents types. On en déduit alors les propriétés les plus caractéristiques des machines et les structures qu'il est nécessaire d'adopter pour en extraire leurs performances optimales.

La présente étude précise un certain nombre de concepts dégagés antérieurement [2]. Par le caractère restrictif des approximations et par le traitement adopté (limité aux aspects globaux) elle ne représente elle-même qu'une introduction à des études plus détaillées en cours.

2. L'interaction rotor-stator dans les machines électriques. Rappel du problème et notations. - Dans toute machine électrique tournante, il est possible de trouver par la pensée au moins une surface $S$ de révolution autour de l'axe de rotation :

- qui soit plongée dans le vide,

- qui soit fixe simultanément par rapport aux référentiels du rotor et du stator,

- qui constitue une séparation des volumes du rotor et du stator.

C'est sur cette surface, appelée surface d'entrefer, que sera défini le couplage rotor/stator à l'aide du tenseur de Maxwell. Il faut noter qu'en général, il existe de nombreuses surfaces correspondant à la définition ci-dessus; elles sont toutes équivalentes.

Les machines seront représentées dans un référentiel cylindrique $(0, r, \theta, z)$, l'axe de rotation étant porté par $0 z$ (voir Fig. 1).

Les champ et induction magnétiques étant notés dans tout l'espace par $\mathbf{H}$ et $\mathbf{B}$, on utilisera fréquemment la valeur de ces grandeurs sur la surface $S$, par l'intermédiaire de leurs deux projections :

$B_{\mathrm{N}}=$ B.n (composante normale à $S$ ),

$H_{\mathrm{T}}=\mathbf{H} \cdot \frac{\mathbf{u} \wedge \mathbf{r}}{|\mathbf{u} \wedge \mathbf{r}|} \begin{aligned} & \text { (composante tangente à un } \\ & \text { parallèle de } S \text { ), }\end{aligned}$

n : vecteur unitaire normal à $S$, orienté vers l'extérieur,

u : vecteur unitaire supportant $\mathrm{O} z$,

$\mathbf{r}:$ vecteur d'espace d'origine $O$.

Alors, on montre que le couple appliqué par le stator sur le rotor s'écrit :

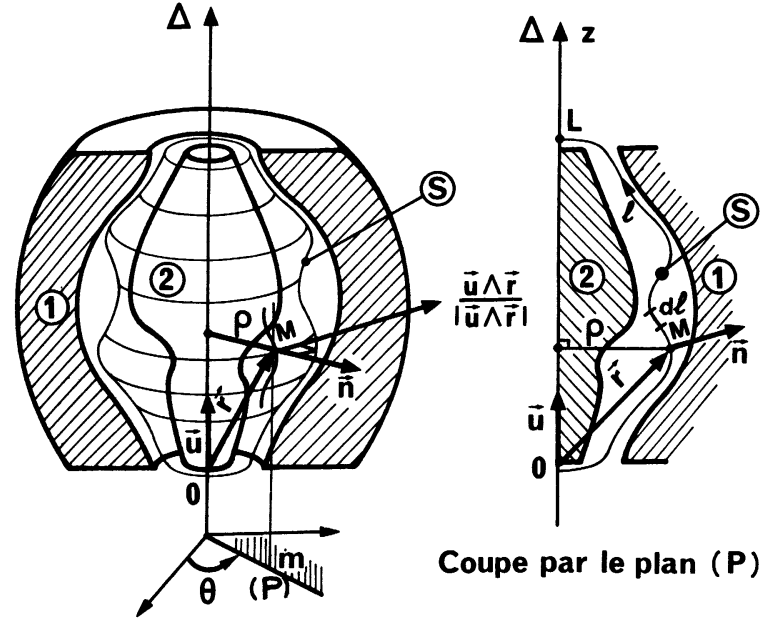

Fig. 1. - Représentation schématique d'une machine tournante $(S)$ : surface d'entrefer.

[Scheme of a rotating machine; $(S)$ : air-gap surface.]

$$
\begin{array}{|l}
C_{12}=\int_{S} B_{\mathrm{N}} H_{\mathrm{T}} \rho \mathrm{d} S \\
\rho \quad=|\mathbf{u} \wedge \mathbf{r}| \text { distance à l'axe de rotation. }
\end{array}
$$

La surface $S$ étant de révolution, paramétrons la position d'un point quelconque $M$ par :

$$
\begin{aligned}
& \text { - l'angle } \theta \text {, } \\
& \text { - l'abscisse curviligne } l \text { mesurée le long d'un } \\
& \text { méridien. }
\end{aligned}
$$

Le système étant cylindrique par rapport à la variable $\theta$, on est incité à décomposer $B_{\mathrm{N}}=B_{\mathrm{N}}(l, \theta)$ et $H_{\mathrm{T}}=H_{\mathrm{T}}(l, \theta)$ en série de Fourier sur $\theta$. On obtient :

$$
\begin{aligned}
& B_{\mathrm{N}}(l, \theta)=\sum_{\alpha \in \mathrm{Z}} \overline{B_{\mathrm{N} \alpha}(l)} \mathrm{e}^{j \alpha \theta} \\
& \left(\operatorname{avec} \overline{B_{\mathrm{N} \alpha}(l)}=\frac{1}{2 \pi} \int_{0}^{2 \pi} B_{\mathrm{N}}(l, \theta) \mathrm{e}^{-j \alpha \theta} \mathrm{d} \theta\right) \\
& H_{\mathrm{T}}(l, \theta)=\sum_{\alpha \in Z} \overline{H_{\mathrm{T} \alpha}(l)} \mathrm{e}^{j \alpha \theta} \\
& \mathrm{Z}: \text { ensemble des entiers relatifs } \\
& \text { (les grandeurs physiques complexes sont sur- } \\
& \text { lignées). }
\end{aligned}
$$

En portant les relations (2) dans (1), on a :

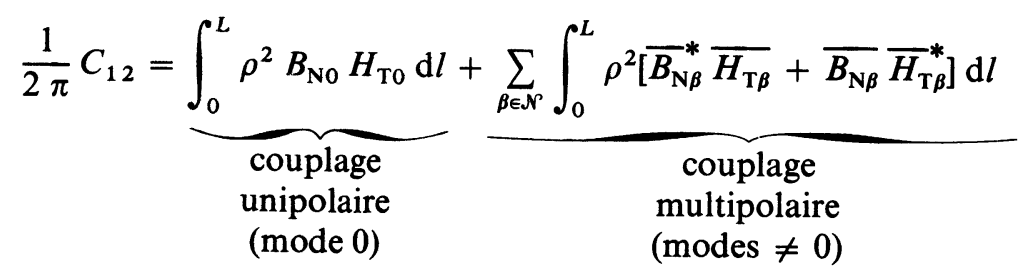

* : conjugaison complexe

$L$ : longueur totale d'un méridien de $S$

$\mathcal{N}$ : ensemble des entiers positifs. 
On voit sur (3) que chaque harmonique d'ordre $\beta \in \mathcal{N}$ participe au couplage électromécanique de manière indépendante des autres.

3. Distribution des champs dans le vide. - Considérons un ensemble quelconque de courants de la forme :

$$
\mathbf{i}=\mathbf{i}(\mathbf{r})=\mathbf{i}(\rho, \theta, z) .
$$

Par décomposition en série de Fourier, on peut écrire :

$$
\begin{aligned}
\mathbf{i} & =\mathbf{i}(\rho, \theta, z)=\sum_{\alpha \in \mathbb{Z}} \overline{\mathbf{i}_{\alpha}(\rho, z)} \mathrm{e}^{j \alpha \theta} \\
& \left.=\mathbf{i}_{0}(\rho, z)+\sum_{\beta \in \mathcal{N}} \overline{\left[\mathbf{i}_{\beta}(\rho, z)\right.} \mathrm{e}^{j \beta \theta}+\overline{\mathbf{i}_{\beta}(\rho, z)^{*}} \mathrm{e}^{-j \beta \theta}\right] \\
& =\underbrace{\mathbf{i}_{0}(\rho, z)+\sum_{\text {multipolaires (réelles) }}}_{\text {unipolaire }} .
\end{aligned}
$$

Dans le vide (perméabilité $\mu=\mu_{0}$ ), le champ $\mathbf{H}$ est noté $\mathbf{H}_{0}$; il est défini par l'intermédiaire des équations de Maxwell :

$$
\mid \begin{aligned}
& \boldsymbol{\nabla} \wedge \mathbf{H}_{0}=\mathbf{i} \\
& \nabla . \mathbf{H}_{0}=0
\end{aligned}
$$

Si nous supposons que les courants sont isolés (conditions aux limites nulles à l'infini), $\mathbf{H}_{0}$ est donné par la forme intégrale :

$$
\mathbf{H}_{0}=\mathbf{H}_{0}(\rho, \theta, z)=\mathbf{H}_{0}(\mathbf{r})=\int_{R^{3}} \mathbf{i}(\mathbf{R}) \wedge \frac{\mathbf{R}-\mathbf{r}}{|\mathbf{R}-\mathbf{r}|^{3}} \mathrm{~d} R^{3}
$$

- R position de chaque volume d'intégration $\left(\mathrm{d} R^{3}\right)$.

Par analogie avec les expressions (4), on peut définir sur $\mathbf{H}_{0}$ les grandeurs

$$
\overline{\mathbf{H}_{0 \beta}}=\overline{\mathbf{H}_{0 \beta}(\rho, z)} \text { et } \quad \mathbf{H}_{0 \beta}=\mathbf{H}_{0 \beta}(\rho, \theta, z) .
$$

Les équations de Maxwell étant linéaires, on a aussi :

$$
\mathbf{H}_{0 \beta}(\mathbf{r})=\int_{R^{3}} \mathbf{i}_{\beta}(\mathbf{R}) \wedge \frac{\mathbf{R}-\mathbf{r}}{|\mathbf{R}-\mathbf{r}|^{3}} \mathrm{~d} R^{3} .
$$

Par suite, à chaque mode $\beta$-polaire de i correspond un mode $\beta$-polaire de $\mathbf{H}$ qui se calcule directement à partir de $\mathbf{i}_{\boldsymbol{\beta}}$.

Remarques : - Comme $\nabla . \mathbf{i}=0$ et $\nabla \cdot \mathbf{H}_{0}=0$, les trois coordonnées de $\mathbf{i}$ et $\mathbf{H}$ ne sont pas indépendantes. En fait, la donnée de deux des coordonnées cylindriques de $\mathbf{i}$ et $\mathbf{H}$ suffisent pour définir complètement ces vecteurs.

- Seuls les termes sources

$$
\mathbf{H}_{0 \beta}=\overline{\mathbf{H}_{0 \beta}} \mathrm{e}^{j \beta \theta}+{\overline{\mathbf{H}_{0 \beta}}}^{*} \mathrm{e}^{-j \beta \theta}
$$

réels ont une réalité physique et peuvent être effectivement mis en œuvre à l'aide d'une densité de courant $\mathbf{i}_{\beta}$ convenablement choisie. Les termes $\overline{\mathbf{H}_{0 \beta}}$ ne sont que des intermédiaires mathématiques.

4. Distributions modales des champs dans un matériau magnétique non saturable. - Considérons un dispositif comprenant à la fois des conducteurs (sièges des courants i) et des matériaux magnétiques. Admettons de plus que la perméabilité locale est scalaire. Les équations de Maxwell définissant $\mathbf{H}$ sont :

$$
\mid \begin{aligned}
& \nabla \wedge \mathbf{H}=\mathbf{i} \\
& \nabla \cdot(\mu \mathbf{H})=0 .
\end{aligned}
$$

En portant (5) dans (8), on peut écrire aussi :

$$
\nabla \wedge\left(\mathbf{H}-\mathbf{H}_{0}\right)=\mathbf{0} .
$$

A partir de (9), on peut définir un potentiel magnétique $V=V(\mathbf{r})$ tel que :

$$
\mid \begin{aligned}
& \mathbf{H}=\mathbf{H}_{0}+\nabla V \\
& V(\mathbf{r})=0 \text { pour } \mathbf{r} \rightarrow 0 .
\end{aligned}
$$

L'équation de $V$ issue de la $2^{\text {e }}$ des expressions (8) s'écrit alors :

$$
\mid \begin{aligned}
& \Delta V+\nabla \chi \cdot\left(\nabla V+\mathbf{H}_{0}\right)=0 \\
& \operatorname{avec} \chi=\log \mu / \mu_{0} .
\end{aligned}
$$

L'équation (11) appelle divers commentaires :

- Elle est linéaire en $V$ et $\mathbf{H}_{0}$. En particulier, si $\mathbf{H}_{0}=\mathbf{0}$, les conditions aux limites donnent $V=0$. Les solutions de $V$ sont des sommes linéaires des solutions des divers monomodes $\mathbf{H}_{0 \beta}$. 
- Elle est très fortement dépendante de $\chi$, en ce sens que dès que $\chi \geqslant 3$ ou 4 , il existe des régions de l'espace pour lesquelles

$$
|\nabla V| \gg \operatorname{Sup}\left|\mathbf{H}_{0}\right| .
$$

Pour étudier (11), nous allons effectuer également une décomposition en série de Fourier de $\chi$. Toujours par analogie avec (4), nous définirons $\bar{\chi}_{\beta}=\bar{\chi}_{\beta}(\rho, z)$ et $\chi_{\beta}=\chi_{\beta}(\rho, \theta, z)$.

Alors l'équation (11) prend la forme générale :

$$
\begin{aligned}
\sum_{n}\left(\Delta_{\theta}-\frac{n^{2}}{\rho^{2}}\right) \bar{V}_{n} \mathrm{e}^{j n \theta}=\sum_{m} \sum_{n}\left(\frac{\partial \bar{\chi}_{m}}{\partial \rho} \cdot \frac{\partial \bar{V}_{n}}{\partial \rho}\right. & \left.-\frac{m n}{\rho^{2}} \bar{\chi}_{m} \bar{V}_{n}+\frac{\partial \bar{\chi}_{m}}{\partial z} \frac{\partial \bar{V}_{n}}{\partial z}\right) \mathrm{e}^{j(m+n) \theta} \\
& +\sum_{m} \sum_{l}\left(\frac{\partial \bar{\chi}_{m}}{\partial \rho} \cdot \bar{H}_{\rho l}+j \cdot \frac{m}{p} \bar{\chi}_{m} \cdot \bar{H}_{\theta l}+\frac{\hat{c} \bar{\chi}_{m}}{\hat{c} z} \bar{H}_{z l}\right) \mathrm{e}^{j(m+l) \theta}
\end{aligned}
$$

$$
\begin{gathered}
\text { avec }: \Delta_{\theta}=\frac{\partial^{2}}{\partial \rho^{2}}+\frac{1}{\rho} \frac{\partial}{\partial \rho}+\frac{\partial^{2}}{\partial z^{2}} \\
l, m, n \in \mathrm{Z}
\end{gathered}
$$

L'équation (12) montre qu'il existe dans le système de nombreux couplages de modes. Comme le système est linéaire par rapport au terme source $\mathbf{H}_{0}$, la solution $V$ générale sera la somme des contributions à $V$ de chaque mode $\overline{\mathbf{H}}_{0 l}$. (Ne pas oublier que $\overline{\mathbf{H}}_{0(-l)}=\overline{\mathbf{H}}_{0 l}{ }^{*}$ pour toute excitation réelle.) Pour simplifier l'étude, nous n'allons considérer dans un premier temps que des termes sources monomodes $\left(\bar{H}_{0 l} \neq 0\right.$ pour une seule valeur $l=l_{0}$ ).

a) Tout d'abord, si $\chi$ est monomode d'ordre zéro $\left(\bar{\chi}_{(m=0)} \neq 0, \chi_{(m \neq 0)}=0\right)$, on voit que $\bar{V}_{n} \neq 0$ si, et seulement si, $n=l_{0}$. Par suite, une distribution de matériaux magnétique complètement de révolution engendre une distribution de $V$ qui reproduit uniquement les modes de $\mathbf{H}_{0}$.

b) Si $\chi$ est monomode d'ordre $m_{0} \neq 0\left(\bar{\chi}_{\left(m=m_{0}\right)} \neq 0\right.$, $\left.\bar{\chi}_{\left(m \neq m_{0}\right)}=0\right)$, le spectre de $V$ contient de nombreux termes non nuls. En effet, le second terme du $2^{\mathrm{e}}$ membre de (12) engendre $\bar{V}_{n} \neq 0$ pour $n=l_{0}+m_{0}$. Ensuite, par récurrence, le premier terme du $2^{\mathrm{e}} \mathrm{mem}$ bre de (12) engendre :

$$
\mid \begin{aligned}
& \bar{V}_{\left\{n+(p+1) m_{0}\right\}} \neq 0 \text { si } \bar{V}_{\left(n+p m_{0}\right)} \neq 0 \\
& \text { avec } p \in \Lambda^{\prime}+\{0\} .
\end{aligned}
$$

Il en résulte que $\bar{V}_{n} \neq 0$ si $n=l_{0}+p m_{0}(p \in \mathcal{N})$.

c) Si $\chi$ est une distribution périodique d'ordre $m_{0}$ (cas très fréquent), alors $\bar{\chi}_{\left(m=p m_{0}\right)} \neq 0$ et $\chi_{\left(m \neq p m_{0}\right)}=0$. Par le même raisonnement que ci-dessus, on obtient de proche en proche le même spectre :

$$
\bar{V}_{n} \neq 0 \quad \text { si } n=l_{0}+p m_{0} .
$$

Si nous rappelons maintenant que $\bar{\chi}_{\left(-\left|m_{0}\right|\right)}=\bar{\chi}_{\left|m_{0}\right|}^{*}$ et $\overline{\mathbf{H}_{0\left(-\left|l_{0}\right|\right)}}={\overline{\mathbf{H}_{0} l_{0}}}^{*}$ dans les cas réels, on voit qu'une excitation de mode $\mathbf{H}_{0\left(l=\left|l_{0}\right|\right)} \neq 0$ et une distribution de perméabilité $\chi_{\left(m=\left|m_{0}\right|\right)} \neq 0$ engendreront tous les modes complexes de la forme $n= \pm\left|l_{0}\right| \pm p\left|m_{0}\right|$. Enfin, si les termes sources sont également réels et périodiques de mode fondamental $\left|l_{0}\right|$, on obtient tous les modes complexes :

$$
n= \pm p^{\prime}\left|l_{0}\right| \mp p\left|m_{0}\right| \text { avec } p, p^{\prime} \in \mathrm{N}+\{0\}
$$

5. Les perturbations de perméabilité. - Notons que dans tout système ferro-magnétique, on a :

$$
\chi=\log \frac{\mu}{\mu_{0}} \geqslant 0 .
$$

Par suite le terme $\chi_{0}$ ne peut jamais s'annuler. Dans la grande majorité des cas concrets (structure magnétique de la machine globalement de révolution), il est même prépondérant. On peut donc le considérer comme la distribution magnétique de base à partir de laquelle les modes d'ordre plus élevé seront considérés comme des perturbations.

Le potentiel magnétique de base $V=V_{0}$ obéira à l'équation :

$$
\Delta V_{0}+\nabla \chi_{0} \cdot\left(\nabla V_{0}+\mathbf{H}_{0}\right)=0 .
$$

Posons d'autre part :

$$
\begin{aligned}
& \chi=\chi_{0}+\chi^{\prime} \\
& V=V_{0}+V^{\prime} .
\end{aligned}
$$

On en tire :

$\frac{\Delta V^{\prime}+\nabla \chi_{0} \cdot \nabla V^{\prime}+\nabla \chi^{\prime} \cdot\left(\nabla V_{0}+\mathbf{H}_{0}\right)}{1^{\mathrm{e}} \text { ordre }}+\frac{\nabla \chi \cdot \nabla V^{\prime}=0}{2^{\mathrm{e}} \text { ordre }}$.

- Lorsque $\chi^{\prime}$ est suffisamment petit (perturbations de perméabilité faibles), le $1^{\mathrm{er}}$ membre de l'équation (14) se réduit au $1^{\mathrm{er}}$ ordre. Alors, les solutions $V^{\prime}$ varient linéairement avec $\chi^{\prime}$; si $\mathbf{H}_{0}$ est monomode d'ordre $\left|l_{0}\right|$, les solutions $V^{\prime}$ reproduisent le spectre de $\chi^{\prime}$ avec un décalage $\pm\left|l_{0}\right|$

- Lorsque la perturbation est assez forte pour que 
les termes du $2^{e}$ ordre de (14) ne soient plus négligeables, la solution est plus complexe, puisqu'elle se

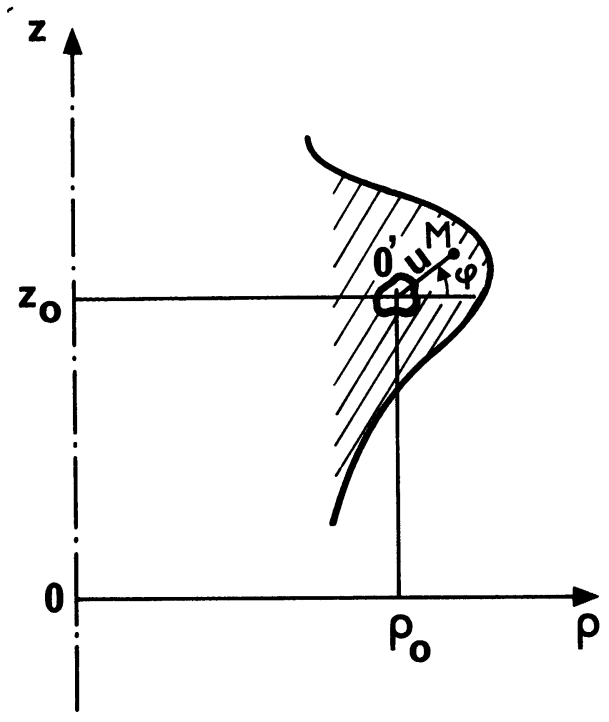

Fig. 2. - Système de référence local pour l'étude de la portée des perturbations (approximation $u \ll \rho_{0}$ ).

[Local system of reference used for the study of the perturbations range (in the approximation $u \ll \rho_{0}$ ).] rapproche du cas général étudié en (12). En supposant à nouveau que $\mathbf{H}_{0}$ est monomode d'ordre $\left|l_{0}\right|$, il existe des solutions de $V^{\prime}$ pour lesquelles le mode $n= \pm\left|l_{0}\right| \pm 0 .\left|m_{0}\right|= \pm\left|l_{0}\right|$ est non nul; cela signifie qu'une perturbation de perméabilité de mode $\left|m_{0}\right| \neq\left|l_{0}\right|$ peut engendrer des perturbations sur la distribution de base du mode $\left|l_{0}\right|$. Dans ce dernier cas, la présence des cavités de modes élevés a pour effet de changer la perméabilité apparente globale (mode 0) du matériau magnétique.

Dans la suite de l'étude, nous allons supposer que les perturbations de perméabilité à partir du mode $\chi_{0}$ seront toujours $d u$ premier ordre, ce qui permettra alors d'additionner directement leurs effets (les perturbations de champ au niveau des entrefers seront donc également additionnables).

6. Portée des perturbations. - Considérons une perturbation de perméabilité $\chi^{\prime}$ de faible valeur, superposée à $\chi_{0}$. Cette perturbation va engendrer une distribution de potentiel $V^{\prime}$ donnée par (14), qui pourra être approximée au $1^{\mathrm{er}}$ ordre. Pour tous les couples $\mathbf{H}_{0 l}$ et $\chi_{m}$, le potentiel $\bar{V}_{(l, m)}^{\prime}$ engendré obéira à la nouvelle équation simplifiée issue de (12) :

$$
\begin{aligned}
& \left\{\begin{array}{l}
\frac{\partial^{2} \bar{V}^{\prime}}{\partial \rho^{2}}+\left(\frac{1}{\rho}+\frac{\partial \chi_{0}}{\partial \rho}\right) \frac{\partial \bar{V}^{\prime}}{\partial \rho}-\frac{(m+l)^{2}}{\rho^{2}} \bar{V}^{\prime}+\frac{\partial \chi_{0}}{\partial z} \cdot \frac{\partial \bar{V}^{\prime}}{\partial z}+\frac{\partial^{2} \bar{V}^{\prime}}{\partial z^{2}} \\
+\frac{\partial \bar{\chi}_{m}}{\partial \rho} \cdot \overline{H_{0 \rho l}^{\prime}}+j \frac{m}{\rho} \overline{\chi_{m}} \cdot \overline{H_{0 \theta l}^{\prime}}+\frac{\partial \bar{\chi}_{m}}{\partial z} \cdot \overline{H_{0 z l}^{\prime}} \\
\operatorname{avec} \mathbf{H}_{0}^{\prime}=\mathbf{H}_{0}+\nabla V_{0} .
\end{array}\right\}=0
\end{aligned}
$$

Supposons maintenant que cette perturbation soit non nulle seulement dans une région $\left(\vartheta_{0}\right)$ bornée du plan $(\rho, z)$ (voir Fig. 2). Hors de $v_{0}$, l'équation (15) se réduit à la $1^{\text {re }}$ ligne du $1^{\text {er }}$ membre. Pour l'étudier, nous allons supposer que :

- les gradients de $\chi_{0}$ sont faibles au voisinage de $\vartheta_{0}$,

$-(m+l)$ est grand.

Si nous prenons pour origine des coordonnées le centre $O$ de $\vartheta_{0}$, l'expression simplifiée de (15) prend la forme :

$$
\mid \begin{aligned}
& \frac{\partial^{2} V^{\prime}}{\partial u^{2}}+\frac{1}{u} \frac{\partial V^{\prime}}{\partial u}+\frac{1}{u^{2}} \frac{\partial^{2} V^{\prime}}{\partial \varphi^{2}}-\frac{(m+l)^{2}}{\rho_{0}^{2}} V^{\prime}=0 \\
& \text { avec } \mid \begin{array}{l}
u=\sqrt{\left(\rho-\rho_{0}\right)^{2}+\left(z-z_{0}\right)^{2}} \\
\varphi=\operatorname{Arctg} \frac{z-z_{0}}{\rho-\rho_{0}}
\end{array}
\end{aligned}
$$

Les solutions de (16) sont constituées par la somme de solutions à variables séparées de la forme [3] :

avec

$$
\bar{V}^{\prime}=\bar{V}^{\prime}(u, \varphi)=\left[\bar{V}_{p}^{\prime}(u)\right] \cdot \mathrm{e}^{j p \varphi}
$$

$$
\bar{V}_{p}^{\prime}(u) \neq \sqrt{\frac{\pi}{2}} \sqrt{\frac{\rho_{0}}{(m+l) u}} \exp -\left(\frac{(m+l) u}{\rho_{0}}\right)\left[1+\frac{\rho_{0}}{8(m+l) u}\left(4 p^{2}-1\right)+\cdots\right] \overline{\mathcal{U}}^{\prime}(p)
$$

où $\bar{u}^{\prime}(p)$ est une constante caractérisant le sous-mode $p$ considéré ; elle dépend du poids de ce dernier dans la décomposition en fonctions cylindriques de la perturbation située dans $\mho_{0}$. 
Les expressions (17) sont fondamentales; elles montrent que l'influence extérieure d'une perturbation locale de perméabilité décroît très vite avec la distance $u$ (en exponentielle de $(m+l))$; cette décroissance $n$ 'est pas fonction de $p$, c'est-à-dire de l'aspect multipolaire en $(\rho, z)$ de la perturbation. Par suite, toute perturbation de perméabilité d'ordre $(m+l)$ située à une distance d'un observateur supérieure à $\frac{\rho_{0}}{m+l}$ n'engendrera qu'un champ faible au niveau de ce dernier.

D'autre part, pour tout observateur situé hors de la région $\vartheta_{0}$, la perturbation est complètement caractérisée par le spectre $\mathcal{U}_{p}^{\prime}$; l'observateur ne peut savoir si la cause de la perturbation extérieure $V^{\prime}$ est due effectivement à de faibles perturbations de perméabilité ou bien à des causes plus brutales localement, telles que de petites cavités vides de matière situées dans $\vartheta_{0}$ (la connaissance du seul spectre $\bar{U}_{p}^{\prime}$ ne permet pas en effet d'effectuer de choix entre ces différentes causes). De plus, l'interaction rotor-stator ne dépend que des champs sur la surface d'entrefer $S$, c'est-à-dire des spectres $\bar{U}_{p}^{\prime}$ au niveau de cette dernière. La manière dont ces champs ont été produits n'a pas d'importance immédiate. Compte tenu de ces éléments, nous pouvons admettre que la théorie linéarisée pour faibles gradients de perméabilités définie par l'approximation du 1 er ordre de (14) peut être étendue sans erreur aux ensembles de petites cavités qui ne sont pas adhérentes à la surface $s\left({ }^{1}\right)$.

7. Premier classement des machines. - Considérons maintenant de manière séparée les contributions aux champs données par le stator (indicé 1) et par le rotor (indicé 2), en nous plaçant dans le cadre de l'équation (14) approximée au $1^{\text {er }}$ ordre (machines linéarisées).

Les champs sources sont $\mathbf{H}_{01}$ et $\mathbf{H}_{02}$. Ils engendrent respectivement les potentiels d'ordre $0\left(V_{01}\right.$ et $\left.V_{02}\right)$ et les potentiels de perturbations $\left(V_{1}^{\prime}\right.$ et $\left.V_{2}^{\prime}\right)$, définis respectivement par équations (13) et (14). Au niveau de l'entrefer, ces différents potentiels engendrent à leur tour les composantes actives :

$$
\begin{aligned}
& \overline{B_{\mathrm{N} \beta, 1}}, \overline{H_{\mathrm{T} \beta, 1}}, \overline{B_{\mathrm{N} \beta, 1}^{\prime}}, \overline{H_{\mathrm{T} \beta, 1}^{\prime}} \\
& \overline{B_{\mathrm{N} \beta, 2}}, \overline{H_{\mathrm{T} \beta, 2}}, \overline{B_{\mathrm{N} \beta, 2}^{\prime}}, \overline{H_{\mathrm{T} \beta, 2}^{\prime}} .
\end{aligned}
$$

En portant dans (3) les composantes ci-dessus, on obtient :

$$
\frac{1}{2 \pi} C_{12}=\left\{\begin{aligned}
& \int_{0}^{\alpha} \rho^{2}\left(\overline{B_{\mathrm{N} \beta, 1}} * \overline{H_{\mathrm{T} \beta, 2}}+{\overline{B_{\mathrm{N} \beta, 2}}}^{*} * \overline{H_{\mathrm{T} \beta, 1}}+\text { compl. conj. }\right) \mathrm{d} l \\
+ & \int_{0}^{\alpha} \rho^{2}\left(\overline{B_{\mathrm{N} \beta, 1}^{\prime}} * \overline{H_{\mathrm{T} \beta, 2}^{\prime}}+{\overline{B_{\mathrm{N} \beta, 2}^{\prime}}}^{*} \overline{H_{\mathrm{T} \beta, 1}}+\text { compl. conj. }\right) \mathrm{d} l \\
+ & \left.\int_{0}^{\alpha} \rho^{2} \overline{\left(\bar{B}_{\mathrm{N} \beta, 1}^{\prime}\right.} * \overline{H_{\mathrm{T} \beta, 2}}+\overline{B_{\mathrm{N} \beta, 2}^{\prime}} * \overline{H_{\mathrm{T} \beta, 1}}+\text { compl. conj. }\right) \mathrm{d} l \\
+ & \text { termes unipolaires }+ \text { termes carrés } .
\end{aligned}\right.
$$

Les termes carrés ont une somme nulle (ils expriment l'interaction sur lui-même de chaque solide). Les termes unipolaires ne sont pas considérés ici.

A partir de (19), on peut effectuer un premier classement, simple, des machines :

- la première ligne correspondra aux machines dites classiques,

- la deuxième ligne aux machines à réluctance variable,

- la troisième aux machines mixtes.

( ${ }^{1}$ Cette extension de la théorie revient à dire que pour toute échelle de longueur grande devant la dimension des cavités, tout matériau magnétique continu et homogène mais percé de trous peut être confondu avec un matériau entièrement continu mais inhomogène. On peut alors remplacer les gradients continus de perméabilité par des distributions de petites cavités convenablement réparties dans un matériau homogène.
Il faut remarquer que les trois types de machines sont semblables en ce qui concerne leur morphologie globale (donnée par le terme prépondérant $\chi_{0}$ ); de même, les distributions de champ peuvent être voisines. Par contre, c'est l'utilisation du champ global qui est différente. Dans les machines classiques, il est utilisé directement pour produire le couple. Dans une machine à réluctance, le couple s'obtient par l'utilisation intermédiaire des anisotropies $\chi^{\prime}$ (modes $\neq 0$ ).

8. Distributions des courants et des cavités dans les deux types de machines. - Pour préciser les conditions de couplage rotor-stator, effectuons une transformation d'intégrale de surface en intégrale de volume à partir de la relation (1) (pour cela, on multiplie l'intégrale par $\mathrm{e}^{x}=\mu / \mu_{0}$, qui est égal à 1 sur la surface). On obtient la détermination usuelle : 
$C_{12}=\int_{\mho(S)}\left(\mathbf{B} \wedge \mathbf{i}+\frac{\mathbf{B} . \mathbf{H}}{2} \cdot \nabla \chi\right)$ ru d

V(S) : volume intérieur à $S$ (rotor) .

Pour le couplage classique, qui ne fait intervenir que $\chi_{0}$, on a $: \nabla \chi_{0}$ ru $=0$. Dans ces conditions, le couplage ne peut être non nul que si des courants circulent dans le rotor. Inversement, pour $\chi^{\prime} \neq 0$, on peut obtenir un couplage par réluctance sans courants dans un des deux solides rotor ou stator.

Pour le couplage classique, où $\chi^{\prime}$ n'intervient pas, la production du couple est associée à des pertes Joule. Pour obtenir un fort couple et de faibles pertes, on est amené à rechercher des matériaux à la fois très magnétiques ( $\mu$ grand) et bons conducteurs. Comme de tels matériaux n'existent pas naturellement, on les crée artificiellement par une disposition composite, où les conducteurs sont placés dans des cavités situées à l'intérieur des matériaux magnétiques. Pour cette raison, les machines à couplage classique comportent donc finalement aussi des termes $\chi^{\prime} \neq 0$.

Par suite, les machines classiques et à réluctance ont des structures globales assez voisines. Dans les deux blocs de matériaux magnétiques qui constituent le rotor et le stator, on perce des cavités.

- Pour un couplage classique, ces cavités contiennent les conducteurs créateurs des champs $\overline{\left.B_{\mathrm{N} \beta,(1} \text { ou } 2\right)}$ et $\overline{\left.H_{\mathrm{T} \beta,(1} \text { ou } 2\right)}$.

- Pour un couplage à réluctance, certaines cavités restent vides et servent simplement à créer les termes $\chi^{\prime}$ non nuls permettant le couplage.

9. Le concept de zone active. Expressions comparées des couples. - D'après l'expression (20), tous les éléments de volume dV peuvent apporter une contribution significative au couple $C_{12}$. En fait, il n'en est rien car, d'après (1) et (17), toute perturbation de perméabilité de mode $\beta$ située à une distance de l'entrefer supérieure à $\rho_{0} / \beta$ ne participe pratiquement pas au couplage. Par analogie, toute distribution de courant $\mathbf{i}_{\alpha}$ de mode $\alpha$ située à une distance de l'entrefer supérieure à $\rho_{0} / \alpha$ est également sans influence sur le couple.

Il en résulte que si les modes prépondérants de $\mathbf{i}$ et $\chi^{\prime}$ sont respectivement $\alpha$ et $\beta$, on peut distinguer de part et d'autre de l'entrefer deux zones d'épaisseurs $\rho_{0} / \alpha$ et $\rho_{0} / \beta$ que nous appellerons (voir Fig. 3) :

- zone active des courants,

- zone active de la réluctance.

A partir de (20) et du concept de zone active, on peut évaluer l'ordre de grandeur du couplage rotor/stator.

Considérons tout d'abord des machines monoentrefers.

Pour le couplage classique les machines ne comportent pas de zones actives de réluctance (structure de la Fig. 4a). On peut avoir une bonne idée du couplage en écrivant :

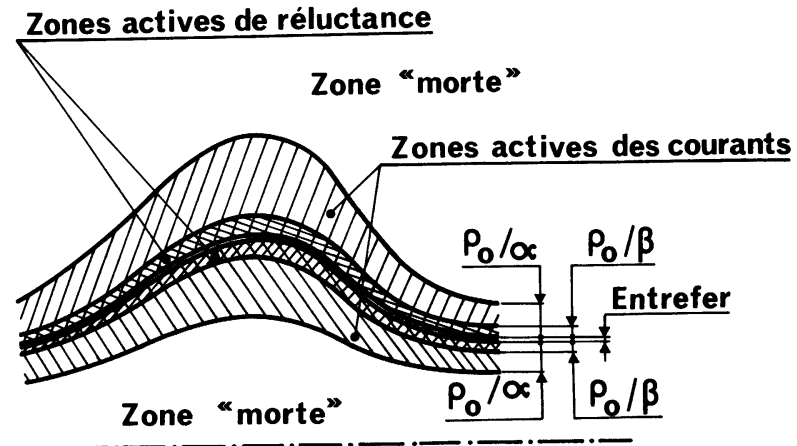

Fig. 3. - Structure générale d'une machine comportant du fer ; délimitation des deux zones actives (courant et réluctance).

[General structure of a machine with iron; boundaries between the two active zones (current and reluctance).]
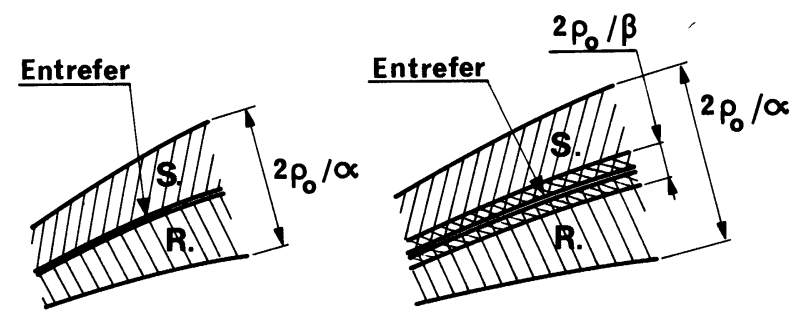

4 a

$4 \mathrm{~b}$
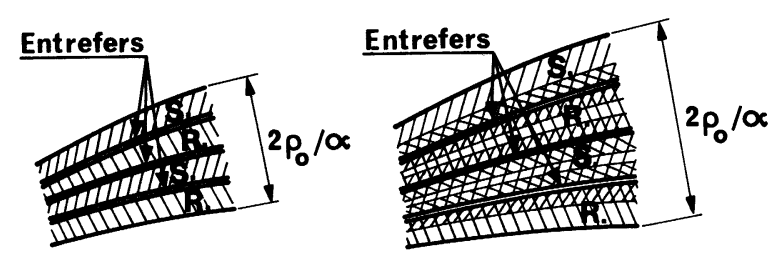

$4 \mathrm{c}$

$4 \mathrm{~d}$

Fig. 4. - Structure des machines classiques et à réluctances. a) Classique monoentrefer ; $b$ ) Réluctance monoentrefer ; $c$ ) Classique polyentrefer; $d$ ) Réluctance polyentrefer.

[Structure of the classical and reluctance machines : a) Classical with one air-gap ; b) Reluctance with one air-gap ; $c$ ) Classical with several air-gaps; d) Reluctance with several air-gaps.]

$$
\cdot \begin{aligned}
C_{12}=\left(C_{12}\right)_{\mathrm{c}}=k_{\mathrm{c}}\langle B\rangle\langle i\rangle & \left\langle\rho_{0}\right\rangle \times \\
& \times\left[\frac{\langle S\rangle\left\langle\rho_{0}\right\rangle}{\alpha}\right]
\end{aligned}
$$

où

- $k_{\mathrm{c}}$ est un coefficient caractéristique de la structure,

- les valeurs entre crochets $〈>$ représentent des valeurs moyennes convenablement définies $\left(\frac{\langle S\rangle\left\langle\rho_{0}\right\rangle}{\alpha}\right.$ représente le volume de la zone active). 
Pour le couplage à réluctance (voir Fig. 4b), on peut écrire par analogie :

$$
\begin{aligned}
C_{12}=\left(C_{12}\right)_{\mathrm{R}}=k_{\mathrm{R}}\langle B\rangle\langle H\rangle\langle|\nabla \chi|\rangle\langle\rho\rangle \times \\
\\
{\left[\frac{\langle S\rangle\left\langle\rho_{0}\right\rangle}{\beta}\right] . }
\end{aligned}
$$

Sachant que $\nabla \chi=\frac{1}{\mu} \nabla \mu$, on remarque que l'équation aux dimensions de $\nabla \chi$ est l'inverse d'une longueur. Donc, pour des distributions de cavités de configuration générale donnée, $|\nabla \chi|$ est proportionnel à $\beta \mid\left\langle\rho_{0}\right\rangle$. On peut alors écrire :

$$
\left(C_{12}\right)_{\mathrm{R}}=k_{\mathrm{R}}^{\prime}\langle B\rangle\langle H\rangle\left[\langle S\rangle\left\langle\rho_{0}\right\rangle\right]
$$

$\langle H\rangle$ : valeur moyenne de $H$ dans la couche d'épaisseur $\rho_{0} / \beta$,

$k_{\mathrm{R}}^{\prime} \quad$ : coefficient caractéristique dépendant à la fois des structures globales (distribution de $B$ ) et locale (distribution des cavités).

Supposons de plus pour simplifier que $\mu / \mu_{0}$ tende vers l'infini dans la couche d'épaisseur $\left(\frac{\rho_{0}}{\alpha}-\frac{\rho_{0}}{\beta}\right)$. Dans ce cas, nous avons :

$$
\langle H\rangle \sim \frac{\langle i\rangle\left\langle\rho_{0}\right\rangle}{\beta} .
$$

L'expression (23) prend la forme nouvelle :

$\left(C_{12}\right)_{\mathrm{R}}=k_{\mathrm{R}}^{\prime \prime}\langle B\rangle \frac{\langle i\rangle\left\langle\rho_{0}\right\rangle}{\beta}\left[\langle S\rangle\left\langle\rho_{0}\right\rangle\right]$.

Pour les machines mixtes :

$-|\beta|=2|\alpha|$,

- les inductions $\langle B\rangle$ et les courants $\langle i\rangle$ sont communs aux deux formes de couplage,

- les deux couples $\left(C_{12}\right)_{\mathrm{C}}$ et $\left(C_{12}\right)_{\mathrm{R}}$ sont égaux et s'alignent sur la valeur la plus faible; en général, on a $k_{\mathrm{c}} \gg k_{\mathrm{R}}^{\prime \prime}$, ce qui nous incite à prendre (25) comme expression du couple $\left({ }^{2}\right)$.

Dans ces conditions, sauf pour quelques usages particuliers que nous n'examinerons pas, les machines mixtes ont un couple inférieur aux machines classiques. Pour simplifier la présentation, nous ne les considérerons plus.

Pour les machines à réluctance où $|\beta| \simeq|\alpha|$, nous obtenons un couplage proche de celui des machines mixtes. Par contre, si $|\beta| \gg|\alpha|$, la situation se modifie profondément. En effet, comparons deux machines, classique et à réluctance, pour lesquelles $\langle B\rangle$ et

$\left(^{2}\right)$ On admettra ici que $k_{\mathrm{c}} \gg k_{\mathrm{R}}^{\prime \prime}$ sans démonstration. Cette inéquation est largement justifiée dans la grande majorité des cas concrets; des démonstrations plus générales seront exposées ultérieurement. $\langle i\rangle$ soient du même ordre. Si ces machines sont constituées par des conducteurs de même nature, les pertes Joule seront voisines. Or, d'après (21) et (25), nous pourrons écrire :

$$
\frac{\left(C_{12}\right)_{\mathrm{C}}}{\left(C_{12}\right)_{\mathrm{R}}}=\frac{k_{\mathrm{c}}}{k_{\mathrm{R}}^{\prime \prime}} \cdot \frac{\beta}{\alpha} .
$$

L'examen du $2^{\mathrm{e}}$ membre de (26) montre que quels que soient $k_{\mathrm{c}}$ et $k_{\mathrm{R}}^{\prime \prime}$, il existe une valeur du rapport $\beta / \alpha$ à partir de laquelle le couple par réluctance devient supérieur au couple classique. Cette propriété fondamentale est liée à la variance différente des expressions (21) et (25) vis-à-vis du coefficient sans dimensions $\beta$, qui peut varier dans de larges limites au point de vue constructif.

10. La saturation dans les machines. Couples maximaux. - L'expression (26), établie en l'absence de saturation, montre que le rendement des machines à réluctance variable est supérieur à celui des machines classiques dès que $\beta / \alpha$ est assez grand. Il en est de même de la puissance volumique si les densités de courant limites sont bornées à des valeurs assez faibles. Par contre, si les densités de courant peuvent être accrues, on observe une saturation du couple beaucoup plus rapidement sur les machines à réluctance que sur les machines sans fer. $\mathscr{S}$ rosso modo, le couple maximal qu'il est possible d'extraire d'une machine classique sans saturer exagérément ses matériaux magnétiques est donné par l'expression classique :

$$
\left(C_{12}\right)_{\mathrm{C}_{\text {moyen }}} \leqslant\left(C_{12}\right)_{\mathrm{C}_{\text {moyen max }}}=\int_{S} \frac{1}{16} \frac{B_{\mathrm{s}}^{2}}{\mu_{0}} \cdot \rho \mathrm{d} S
$$

où $B_{\mathrm{s}}$ est l'induction au coude de saturation.

En pratique, sur les turboalternateurs, les couples effectivement atteints sont supérieurs à $50 \%$ du couple moyen maximal défini ci-dessus.

Pour les machines à réluctance variable, nos connaissances actuelles ne sont pas assez avancées pour que nous puissions chiffrer avec précision leurs limitations. Cependant, nous estimons raisonnable l'inéquation ci-dessous [4] :

$$
\begin{aligned}
& \left(C_{12}\right)_{\mathrm{R}_{\text {moyon }}} \leqslant k_{\mathrm{RC}}\left(C_{12}\right)_{\mathrm{C}_{\text {moyen max }}} \\
& \text { avec } k_{\mathrm{RC}} \simeq 0,15 .
\end{aligned}
$$

Comme $k_{\mathrm{RC}} \ll 1$, la comparaison directe de (27) et (28) montre que le couple maximal qu'il est possible d'extraire d'une machine classique est très supérieur à celui d'une machine à réluctance variable ayant une surface d'entrefer analogue.

Toujours en supposant $\beta \gg \alpha$, la considération conjointe des relations (26) et (27)-(28) aboutit aux situations successives suivantes :

- tant que le couple maximal défini par (28) n'est 
pas atteint, les machines classiques ont, pour les mêmes pertes Joule, un couple inférieur à celui des machines à réluctance,

- au prix d'une augmentation sensible des pertes Joule, les machines classiques peuvent à leur tour atteindre le couple limite donné par (28),

- en accroissant encore fortement les pertes, les couples délivrés par les machines classiques peuvent dépasser de beaucoup ceux des machines à réluctance ; si les machines ne sont pas en régime saturé franc, une nouvelle limitation est donnée par (27),

- enfin, au prix d'un nouvel accroissement des pertes, les machines classiques entrent en régime de saturation forte et se rapprochent des machines sans fer, aux densités de puissance limites extrêmement élevées.

La figure 5 représente ces étapes successives.

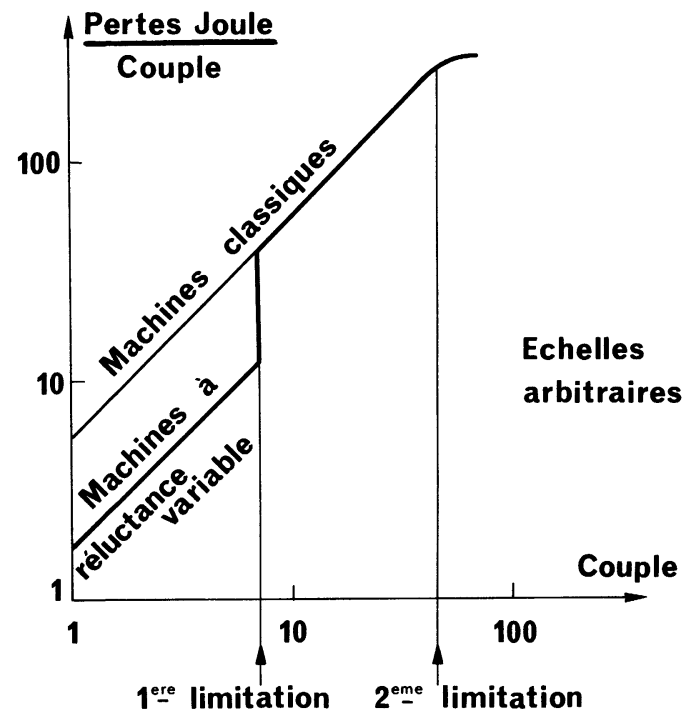

Fig. 5. - Pertes Joule/couple en fonction du couple, pour des machines classiques et à réluctance de dimensions et de surfaces d'entrefer semblables. La première limitation correspond à la relation (28). La deuxième à la relation (27).

[Comparison of the relative losses as a function of the output torque for classical and reluctance machines having similar dimensions.]

Ces situations que nous venons d'analyser sur une machine mono-entrefer entraînent des écarts profonds de morphologie générale sur les deux types de machines, que nous allons examiner ci-après.

11. Machines polyentrefers. Structure générale des machines. - Considérons tout d'abord une machine classique pour laquelle nous disposons dans les deux zones actives $\left(\rho_{0} / \alpha\right), N$ entrefers comme indiqué sur la figure $4 c$. Si nous conservons les mêmes valeurs de $\langle B\rangle$ et $\langle i\rangle$, le couple $C_{12}$ donné par (21) est pratiquement indépendant de $N$. Par contre, le couple limite donné par (27) satisfera l'équation :

$$
\left(C_{12}\right)_{\mathrm{CN}_{\text {moyen }}} \leqslant N\left(C_{12}\right)_{\mathrm{C}_{\text {moyen max }}} .
$$

Il sera augménté pour $N>1$.
Considérons maintenant une machine à réluctance pour laquelle on dispose dans les deux zones actives de courant $\left(\rho_{0} / \alpha\right), N$ entrefers flanqués de zones actives à réluctance $\left(\rho_{0} / \beta\right)$ (Fig. $\left.4 d\right)$.

Le nouveau couple de réluctance vaut, à partir de (23) :

$$
\left(C_{12}\right)_{\mathrm{R} N}=N k_{\mathrm{R}}^{\prime}\langle B\rangle\langle H\rangle\left[\langle S\rangle\left\langle\rho_{0}\right\rangle\right] .
$$

Dans chaque zone active à réluctance, on $\mathrm{a}$, à partir de (24) :

$$
\langle H\rangle \sim \frac{1}{N} \frac{\langle i\rangle\left\langle\rho_{0}\right\rangle}{\beta} .
$$

Il en résulte que $\left(C_{12}\right)_{\mathrm{R} N}$ ne dépend pas de $N$. Par suite, comme pour les machines classiques, on voit que :

- pour des valeurs de $\langle B\rangle$ et $\langle i\rangle$ données, le couplage par réluctance est pratiquement indépendant de $N$.

Parallèlement, le couple maximal vaudra :

$$
\left(C_{12}\right)_{\mathrm{R}_{\text {moyen }}} \leqslant N k_{\mathrm{RC}}\left(C_{12}\right)_{\mathrm{C}_{\text {moyen max }}} .
$$

Il augmentera également comme $N$. Notons cependant que l'équation ci-dessus n'est valable que pour $N \leqslant \beta / \alpha$. Si $N$ croît au-delà de $\beta / \alpha$, il n'y a plus d'augmentation du volume actif de réluctance et donc du couple maximal.

Il en résulte que quel que soit $N($ avec $N \leqslant \beta / \alpha)$, les machines classiques et à réluctance se comportent de la même manière :

- le couple maximal limite croît comme $N$; pour $N$ donné, il est supérieur sur les machines à couplage classique,

- pour un couple donné, les pertes Joule sont indépendantes de $N$ pour chaque type de machine; si leur couple limite n'est pas atteint, les pertes des machines à réluctance sont toujours inférieures à celles des machines classiques.

Si l'on veut réaliser des machines à rendement convenable, on est amené pour les deux types à des choix de la valeur de $N$ opposés.

Pour les machines classiques, avec $N$ égal à 1 , le couple limite est déjà élevé et les pertes Joule importantes. En effet, excepté pour les très grandes puissances (turboalternateurs), les machines classiques qui travaillent près de la valeur $\left(C_{12}\right)_{\mathrm{C}_{\text {moyen max }}}$ ont des rendements assez faibles et posent des problèmes de refroidissement difficiles. Par suite, il est en général peu intéressant de réaliser des machines classiques polyentrefers $\left({ }^{3}\right)$.

Pour les machines à réluctance, où le couple maximal par entrefer est plus faible et le rendement plus élevé,

$\left({ }^{3}\right)$ On montre de plus que les machines classiques polyentrefers non saturées seraient d'ailleurs elles-mêmes supplantées par les machines monoentrefer pour lesquelles $\langle B\rangle \gg B_{\mathrm{s}}$ (1) (c'est-à-dire en pratique par les machines sans fer). 
il est au contraire intéressant d'accroître $N$, car l'accroissement de la puissance conserve des rendements encore suffisamment élevés pour rester tout à fait acceptables. Pour des valeurs $N=\beta / \alpha$ suffisamment élevées, il est même possible de réaliser des machines à réluctance ayant un couple nettement supérieur à $\left(C_{12}\right)_{\mathrm{C}_{\text {moyen max }}}$, avec un rendement encore important.

12. Comparaison des machines. Récapitulation. - Dans le cadre des approximations générales indiquées en introduction, nous venons de montrer que les machines comportant des matériaux magnétiques peuvent se subdiviser en trois classes :

- les machines classiques,

- les machines à réluctance variable,

- les machines mixtes.

Le cas mixte, aux propriétés intermédiaires peu inté- ressantes en général, n'a pas été étudié ici ; ignorons-le.

Formellement, les morphologies des différents types sont semblables :

- le rotor et le stator sont constitués par des masses magnétiques de révolution dans lesquelles on a percé des cavités; ces masses sont séparées par un entrefer de faible épaisseur ;

- dans une partie des cavités sont placés des bobinages créant un champ magnétique global important.

Cependant, le principe même de fonctionnement est profondément différent :

- dans les machines classiques, le couplage électromécanique rotor-stator s'effectue directement à l'aide du champ global,

- dans les machines à réluctance, le couplage est obtenu à l'aide des perturbations au champ local dû à la présence des cavités placées au voisinage de l'entrefer.

Les deux types de couplage se caractérisent par l'introduction de deux échelles de longueur :

- une échelle globale, de valeur

$$
\frac{\rho_{0}}{\alpha}=\frac{\text { rayon moyen de l'entrefer }}{\text { nombre de paires de pôles du bobinage }}
$$

- une échelle locale, de valeur $\left({ }^{4}\right)$

$$
\frac{\rho_{0}}{\beta}=\frac{\text { rayon moyen de l'entrefer }}{\text { ordre de répétition angulaire du motif des cavités }} .
$$

Les performances dépendent essentiellement :

- pour les machines classiques, du rapport $\rho_{0} / \alpha$, - pour les machines à réluctance, des deux rapports $\rho_{0} / \alpha$ et $\beta / \alpha$.

Il en résulte que leurs caractéristiques relatives dépendent du paramètre de construction largement variable $\beta / \alpha$.

Lorsque $\beta / \alpha$ est petit (par exemple $<$ à 5 ou 10 ), les machines à réluctance sont toujours intrinsèquement inférieures aux machines classiques.

Lorsque $\beta / \alpha$ est grand, la situation est plus complexe :

- l'effort limite maximal par unité de surface d'entrefer est plus élevé pour les machines classiques que pour les machines à réluctance,

- si un couple donné peut être obtenu par une

$\left({ }^{4}\right)$ En toute rigueur, il existe pour chaque point de l'entrefer deux échelles de longueurs (globale et locale) déterminées par la distance à l'axe du point considéré. Pour une machine à rotor non cylindrique, les échelles moyennes définies ci-dessus suffisent dans une première approche. 'machine à réluctance, son rendement est supérieur à celui de la machine classique correspondante.

Compte tenu des deux éléments ci-dessus :

- il est peu intéressant d'accroître la surface d'entrefer des machines classiques; ces dernières auront donc une structure simple, monoentrefer,

- il est au contraire très intéressant d'accroître la surface d'entrefer des machines à réluctance (par exemple par des structures polydiscoïdes ou polycylindriques) ; alors, aussi bien leur couple limite que leur rendement peuvent dépasser notoirement ceux des machines classiques habituelles.

13. Conclusion. - L'étude que nous venons d'effectuer constitue une première approche comparative des machines utilisant des matériaux magnétiques. Elle montre l'intérêt, pour des applications différentes, de deux types de machines bien connues :

- les machines classiques,

- les machines à réluctance à grand nombre de dents (l'ordre de répétition du motif angulaire des cavités est grand).

Une suite naturelle à cette étude consisterait à 
calculer les coefficients d'interaction entre les cavités, ce qui permettrait alors d'effectuer des comparaisons quantitatives ; cette question est en cours.
Remerciements. - L'auteur remercie Monsieur Bernard Leroux pour les fructueuses discussions qu'ils ont eues ensemble sur cette étude.

\section{Bibliographie}

[1] Durand, E., Magnetostatique (Masson, Paris) 1968.

[2] Théorie générale comparative des machines électriques établie à partir des équations du champ électromagnétique. Rioux, C., Rev. Gen. Electr. 79 (1970) 415.

[3] Abramovitz, M. and Stegun, I. A., Handbook of Mathematical Functions.
[4] Performances intrinsèques des machines à réluctance variable à disques imbriqués.

Bastos, J. P., Goyet, R. et Lucidarme, J., Revue Phys. Appl. 15 (1980) 45-54. 